result, the skills for helping those with other disorders are

\section{Editor}

Prof. Hamid Ghodse

\section{Editorial board}

Dr JOHn HEN DERSON

Mr DAVE JAGo

Dr N ASSER LOZA

Dr Brian Martin dale

Dr Shekhar Saxena

Prof. David SKuse

Design (c) The Royal College of Psychiatrists 2004.

For copyright enquiries, please contact the Royal College of Psychiatrists.

All rights reserved. No part of this publication may be reprinted or reproduced or utilised in any form or by any electronic, mechanical or other means, now known or hereafter invented, including photocopying and recording, or in any information storage or retrieval system, without permission in writing from the publishers.

The views presented in this publication do not necessarily reflect those of the Royal College of Psychiatrists, and the publishers are not responsible

for any error of omission or fact.

The Royal College of Psychiatrists is a registered charity (no. 228636)

Printed in the UK by Henry Ling Limited at the Dorset Press, D orchester DT1 1HD. challenging in practice, as there are various problems to be

\section{David Skuse}

here seems a certain inevitability that traditional methods of health care delivery are going to change in the age of the internet. To a degree, change is already happening, as specialists around the world share information via broadband links that enable them better to assess and treat patients, for example in the fields of radiology or dermatology, where the presentation of visual information is critical. It is less obvious that specialists would find it valuable to share clinical observations in psychiatry, a specialty where the aural medium of communication is so much more disappearing (Goldberg, 1997). The Royal College of Psychiatrists, psychiatric associations and academic departments of mental health and psychiatry have not really addressed this worrying issue and there are some important consequences, particularly in relation to the training of future general practitioners ( $N$ ey \& Jones, 1985). Traditional learning/teaching still takes place in mental illness institutions in many countries and teaching tends to focus on severe mental disorders, even though those who will become general practitioners are likely to see each year only a few patients with major psychiatric disorders, while they will probably treat depressed and anxious patients every working day. Proposals in some countries for curriculum revision, so that undergraduate teaching concentrates on fundamental concepts and common conditions rather than psychosis, therefore seem particularly pertinent (Working Party of the Education Committee of the Royal College of Psychiatrists, 1997).

across national boundaries are fundamental if the issues relating to undergraduate education and training are to be adequately addressed, worldwide. Psychiatry to day, more than at any other time, is in need of far greater intercollegiate and cross-national collabo ration and cooperation in the psychiatric education of future doctors and in the training of psychiatrists. The initiative of the World Psychiatric Association and the World Federation for Medical Education to develop a core curriculum has undoubtedly been a major step in that direction (Walton $\&$ Gelder, 1999). The World Health 0 rganization, the Royal C ollege of Psychiatrists and its International Regional Groups, as well as national psychiatric associations and academic departments of psychiatry, can and should play their part, too. Although the challenges are considerable, we should not lose sight of the fact that good progress has been made. However, there is still a long way to go.

The apparently logical next step - of teaching psychiatry in the community - although attractive in principle, is overcome, such as how to structure library time, research time, discussion and meeting time with colleagues.

A particular challenge confronting psychiatry is the continuing stigmatisation of mental diso rders. Excellent education of all undergraduates in the subject, to enable all doctors to meet and treat people with mental disorders confidently, is one way to tackle this. But stigmatisation extends beyond the disorders to the specialty itself, and can create a lack of credibility for psychiatry as a whole. This will be remedied only by good departments that offer good teaching and carry out good research, preferably in institutions where the subject is represented at the highest level (i.e. on multi-faculty academic boards).

Academic psychiatry by its very nature cannot be provincial. Collaboration and cooperation between different medical schools within the same country as well as

\section{References}

Ghodse, A. H. (1997) Challenges to academic psychiatry. In The Best and the Worst of Academic Psychiatry - Proceedings of the First European Meeting of the Association of Professors of Psychiatry (eds A. H. Ghodse \& D. Goldberg), pp. 5-7. London: St George's Hospital Medical School.

Goldberg, D. (1997) Academic psychiatry and the changing world. In The Best and the Worst of Academic Psychiatry Proceedings of the First European M eeting of the Association of Professors of Psychiatry (eds A. H. Ghodse \& D. Goldberg), pp. 8-9. London: St George's Hospital Medical School.

Ney, P. G. \& Jones, L. S. (1985) Psychiatry in the medical curriculum. Canadian Journal of Psychiatry, 30, 586-592.

Ring, H., Mumford, D. \& Katona, C. (1999) Psychiatry in the new undergraduate curriculum. Advances in Psychiatric Treatment, 5. $415-419$.

Sierles, F. S. \& Taylor, M. A. (1995) Decline of US medical student career choice of psychiatry and what to do about it. American Journal of Psychiatry, 152, 1416-1426.

Walton, H. \& Gelder, M. (1999) Core curriculum in psychiatry for medical students. M edical Education, 33, 204-211.

Working Party of the Education Committee of the Royal College of Psychiatrists (1997) Core psychiatry for tomorrow's doctors. Psychiatric Bulletin, 21, 522-524.

\title{
Psychiatry and the internet: a new dawn?
}

Behavioural and Brain Sciences Unit, Institute of Child Health, London WC1 1EH, UK, email d.skuse@ich.ucl.ac.uk

important than the visual. We have asked four experts in telemedicine to give us their views on the current status of novel communication technology with special relevance to psychiatry.

Dr Ricky Richardson is the Chairman of the UK eH ealth Association and he gives an upbeat analysis of how clinical practice is likely to change dramatically over the next decade. There is no doubt the UK government is strongly supportive of proposals to use electronic media to increase efficiencies in the $\mathrm{N}$ ational $\mathrm{H}$ ealth Service, but the degree to which inequities in health provision can be addressed by eH ealth initiatives is open to question. A 
more specific discussion of these issues is provided by Professor Peter Yellowlees, a community psychiatrist who has been influential in the development of eH ealth applications in Australian psychiatry. There is obviously merit in using internet-based technology to consult with remote rural communities who lack their own specialist psychiatrist, and the theme is taken up in our third contribution, from $\mathrm{Dr}$ D onald $\mathrm{H}$ ilty and colleagues at the U niversity of C alifornia. They write from a US perspective, and discuss the difficult question of whether telepsychiatry is a cost-efficient way of delivering services. The answer appears to be 'Maybe, in some circumstances'. Finally, Dr Paul McLaren, a consultant psychiatrist from London, brings us back to Europe and cautions that, although professionals like to use videoconference facilities to organise discussions or teaching around clinical matters, there is still resistance to the use of the technology for direct clinical contact. Whether such prejudice will evapo rate as we all become more 'internet literate' is likely to vary according to culture (in every sense of that word).

\title{
eHealth and ePsychiatry for Europe
}

\author{
Ricky J. Richardson
}

Honorary Consultant Paediatrician, Great Ormond Street Hospital for Children, London; Chairman, UK eHealth Association; Chairman, EHTEL eHealth Working Group; Vice-President, International Society for Telemedicine; Clinical Director, HealthSystems Consultants Ltd, 7 Emlyn Road, London W12 9TF, UK, email r.j.richardson@BTinternet.com

\begin{abstract}
he use of the term 'eH ealth' is gathering momentum across Europe as the wider implications of the health care reforms enabled by information technology become apparent. There is a growing realisation by both health care providers and consumers alike that change in the sector is now imperative and timely. Member states of the European Community are all facing an increase in the requirement for health care provision because of changing demography, in particular ageing populations. In the face of an increasing demand for services, the existing model for health care delivery is arguably inadequate. In many ways it is economically unsustainable, even by the wealthiest countries in Europe.

To date, the use of information technology in the European health care sector has been on a pilot basis. $0 \mathrm{n}$ the other hand, we are beginning to see the widespread deployment of managed clinical networks, electronic patient records and other applications made possible by information technology. It is arguable that such applications are now challenging a traditional model of health care delivery that has remained largely unchanged for over 6000 years.

The generic term 'eH ealth' embraces four central pillars of activity. These are:

o clinical applications

O elearning tools

$O$ use of the media

o lifetime health records.
\end{abstract}

\section{Clinical applications}

The first pillar supports an array of clinical applications, which include teleconsultations. These may be arranged in a variety of ways, including interactive videoconferencing (previously known as 'telemedicine', a now obsolete term) or face-to-face consultations via video-links. 0 ther eH ealth applications include software that supports clinical decisionmaking, vital signs monitoring services managed by call centres, telephone-enabled health information (such as the nurse-led service established through N HS D irect in the UK), national eBooking and ePrescription services, home telecare and eN ursing. Few of these facilities are yet applicable to the provision of psychiatric services, but they could become relevant as the infrastructure of eH ealth grows.

\section{eLearning tools}

The second pillar is the use of eLearning to ols to deliver personalised continuing educational programmes to professionals. The traditional, hierarchical health care professional community is being replaced by a more horizontal structure, based around the multi-disciplinary team.

W ithin this evolving structure, there is a different work ethic. Patients are increasingly involved in decision-making about their own clinical management. In the future, the patient will be placed at the centre of the care pathway. In other words, gone are the days when the patient said 'Yes doctor', 'N o doctor', 'I will do what you say doctor' and was grateful for a few minutes of the doctor's valuable time. Patients are becoming informed partners in the health care exchange process: empowered patients know exactly what they want and from where they wish to obtain the service, and if they do not get what they want from one physician, they are willing to go elsew here.

\section{Use of the media}

The third pillar of eH ealth concerns the proactive use of the media - television, radio, newspapers, journals and magazines - to deliver appropriate health care messages to specific segments of the general public. Educating the public about health is no longer the province of public
We are beginning to see the widespread deployment of managed clinical networks, electronic patient records and other applications made possible by information technology. It is arguable that such applications are now challenging a traditional model of health care delivery that has remained largely unchanged for over 6000 years. 